\title{
STRAWBERRY MATURITY NEURAL NETWORK DETECTNG SYSTEM BASED ON GENETIC ALGORITHM
}

\author{
Liming $\mathrm{Xu}{ }^{1, *}$, Yanchao Zhao ${ }^{1}$ \\ ${ }^{1}$ College of Engineering, China Agricultural University, Beijing, P. R. China 100083 \\ * Corresponding author,address: College of Engineering, China Agricultural University, \\ Beijing, 100083,P.R China,Tel:86-10-62737291 Email:xlmoffice@126.com
}

Abstract: The quick and non-detective detection of agriculture product is one of the measures to increase the precision and productivity of harvesting and grading. Having analyzed $\mathrm{H}$ frequency of different maturities in different light intensities, the results show that $\mathrm{H}$ frequency for the same maturity has little influence in different light intensities; Under the same light intensity, three strawberry maturities are changing in order. After having confirmed the $\mathrm{H}$ frequency section to distinguish the different strawberry maturity, the triplelayer feed-forward neural network system to detect strawberry maturity was designed by using genetic algorithm. The test results show that the detecting precision ratio is $91.7 \%$, it takes $160 \mathrm{~ms}$ to distinguish one strawberry. Therefore, the online non-detective detecting the strawberry maturity could be realized.

Key words: $\quad$ genetic algorithm; neural network; maturity; strawberry

\section{INTRODUCTION}

High-quality products not only need the feasible planting management, but also are harvested in the optimum mature period. There are many researches about fruit maturity test by using image processing. The machine vision system (Miller et al., 1989) has been carried to check the fresh peach and confirm the peach maturity by comparing the peach natural colour to the standard hue of different maturity. The broccoli has been classified into no-

Please use the following format when citing this chapter:

$\mathrm{Xu}$, L. and Zhao, Y., 2009, in IFIP International Federation for Information Processing, Volume 294, Computer and Computing Technologies in Agriculture II, Volume 2, eds. D. Li, Z. Chunjiang, (Boston: Springer), pp. 1201-1208. 
ripe, ripe and over-ripe according to the average of special frequency respond(Qiu et al.,1992)。 The colour image processing system has been constructed (Yoshitaka et al., 1997), the test results show that the influence of the quantity of light and the camera angle have no influence on Hue $(\mathrm{H})$ and Saturation (S), $\mathrm{H}$ could be used to distinguished the different maturity banana and tomato. The RGB model has been transformed into HIS model (Choi et al.,1995), the cumulating $\mathrm{H}$ distributing map of fresh tomato was divided into 6 grades, and tomato maturity Index was constructed. The watermelon maturity distinguishing system has been built based on computer vision( Masaru et al.,1997). The green skin stripe image of samples were collected, the RGB model was transformed into the HIS model, the watermelon was divided into three grades: on-ripe, ripe and over-ripe. The maturity, sugar and surface damage of the strawberry has been determinanted by mean of $\mathrm{a}^{*}$ value in $\mathrm{L} \mathrm{a}^{*} \mathrm{~b}^{*}$ model and sugar meter( Masateru et al.,1997).

At present, the Chinese scholars have begun to study the fruit maturity through image procession. The external colour feature from the hue histogram by using computer vision has been extracted to established a three-layer feed-forward neural network system by using genetic algorithm, through which could realize on-line automated detection of apple maturity(Yang et al.,1997). The peach maturity according to the peach feature frequency of impedance has been determined(Ye et al., 1999). the watermelon maturity by its librating frequency respond has been judged (Wang et al.,1999). The external feature by using $\mathrm{H}$ has been distilled to detected automatically the tomato maturity by mean of multilayer feed forward neural network with GA.(Zhang et al.,2001). The change of tomato maturity under different light intensity, presented the maturity detecting foundation has been simulated (Cao et al., 2001). The triple-layer feedforward network to extract colour feature from citrus image has been constructed to show the ratio of total soluble solid (TSS) to titratable acid (TA), then estimate the citrus maturity ( Xu et al., 2001) .The colour and vein of a lot of lack-nutrition leaves image planted by using genetic algorithm has been optimized and chosen to recognize the lack of nutrition (Mao et al.,2003). The non-destructive detecting electron nose according to the apple smell has been built to detect the samples by using main-element analysis method and genetic algorithm network, the results show that the correcting ratio of genetic neural network is higher(Zhao et al.,2004).

For the traditional strawberry, the red fruit surface indicates ripeness. The "Tong-Zi-Yi-Hao" strawberry is different to the traditional strawberry, while the surface is whole red, the fruit is not mature. The colour is changing from light-red, fresh-red to black-red, the black-red strawberry is the optimum maturity. Based on the closed light simulation box, this paper analyzed the changing rule of $\mathrm{H}$ frequency in different light intensity, constructed the 
strawberry neural network detecting system based on genetic algorithm to distinguish its maturity on-line by the fruit image.

\section{MATERIALS AND METHODS}

\subsection{Design of the image information collecting system}

The hardware and software of the image collecting system are same with $\mathrm{Xu}$ et al (2007).

\subsection{Chosen of strawberry maturity distinguishing value}

1) the colour feature value of different strawberry maturities

For every strawberry, the pixel (or frequency) is different, so it could reflect the colour feature. The strawberry colour is mainly red, the colour depth is not different, this paper mainly analyzes the $\mathrm{H}$ hisgram.

In HIS model, the value of $\mathrm{H}$ is from $0^{\circ}$ to $360^{\circ}$. The $\mathrm{H}$ hisgram of different mature strawberries in different light intensities (Fig. 1) were got.

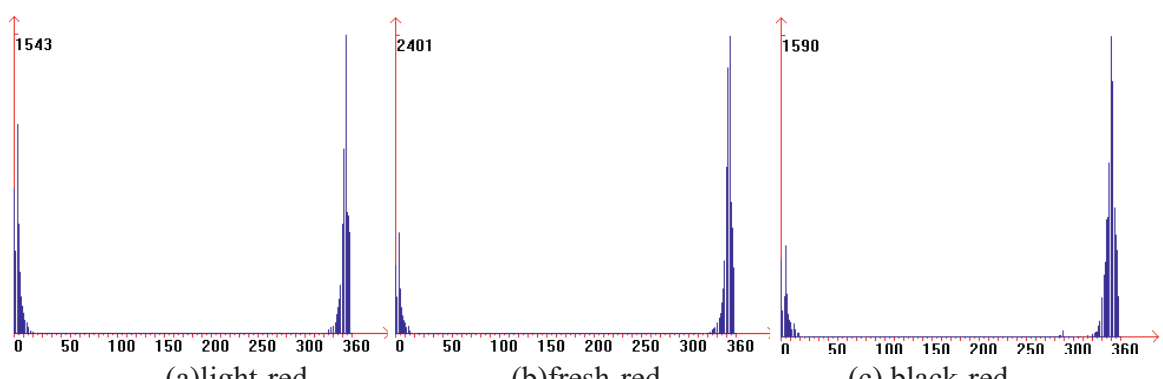

(a)light-red

(b)fresh-red

(c) black-red

Fig. $1 \mathrm{H}$ histgram

In Fig.1, the $\mathrm{H}$ value in different mature strawberry is distributed in two regions, $0^{\circ} \sim 30^{\circ}$ and $330^{\circ} \sim 360^{\circ}$. The $\mathrm{H}$ hisgram comparison of different maturity strawberries in different light intensity is difficult to distinguish the different maturity strawberry. So the statistic of $\mathrm{H}$ hisgram is gained, the distance is $5^{\circ}$, the formula of $\mathrm{H}$ frequency is following:

$\mathrm{Fi}=\left(\right.$ the pixel of $\mathrm{a}^{*}$ per i) /the total pixels in the image

(1) $\mathrm{H}$ frequency of the same strawberry maturity in different light intensities 
The standard light intensities were chosen, they were 8751x, 25101x, $33901 x$, 46601x. In these light intensities, the $\mathrm{H}$ frequency of every strawberry was gained (Fig.2).

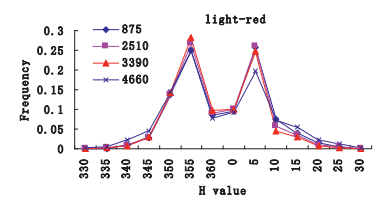

(a)light-red

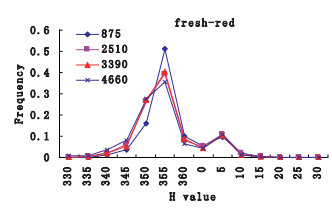

(b)fresh-red

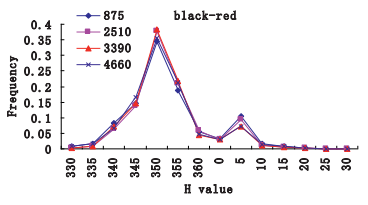

(c)black-red

Fig.2 H frequency of same maturity strawberry in different light

Under the different light intensities, $\mathrm{H}$ frequency of the same maturity strawberry has little change. Therefore, $\mathrm{H}$ frequency is not affected by the light intensity.

For the light-red strawberry, $\mathrm{H}$ frequency has "two-apices" when $\mathrm{H}$ is $355^{\circ}$ and $5^{\circ}$ respectively, the tops are 0.26 and 0.24 respectively.

The $\mathrm{H}$ frequency of the fresh-red strawberry and black-red strawberry is very similitude, there are also two apices, but one is higher, the other is lower. The fresh-red strawberry gets 0.41 higher apices when $\mathrm{H}$ is $355^{\circ}$. The black-red strawberry reaches the 0.37 higher apices when $\mathrm{H}$ is $350^{\circ}$. The two strawberries go down to the lower at 0.1 and 0.09 respectively.

(2) $\mathrm{H}$ frequency of different strawberry maturities in same light intensity

Fig. 3 shows the difference among the different maturities. Under the same light intensity, there are clear differences among different maturities. The $\mathrm{H}$ frequency of different strawberry maturities in the same light intensity forms two sections.

When $\mathrm{H}$ is changing from $330^{\circ}$ to $360^{\circ}$, the black-red strawberry appear the apices at $\mathrm{H}=350^{\circ}$, the light-red strawberry and the fresh-red strawberry appears apices at $\mathrm{H}=355^{\circ}$. The apices of the fresh-red strawberry are higher than that of the light-red strawberry. Under the different light intensities, the apices are different.

When $\mathrm{H}$ is changing from $0^{\circ}$ to $30^{\circ}$, the three strawberry maturities appear the apices at $\mathrm{H}=5^{\circ}$, and the $\mathrm{H}$ frequency is going down in turn. Therefore, this section is able to be used to distinguish the strawberry maturity (Fig.4).
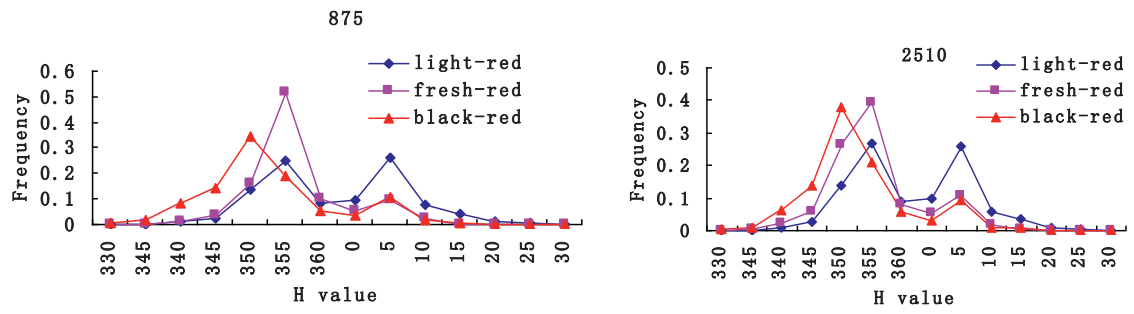

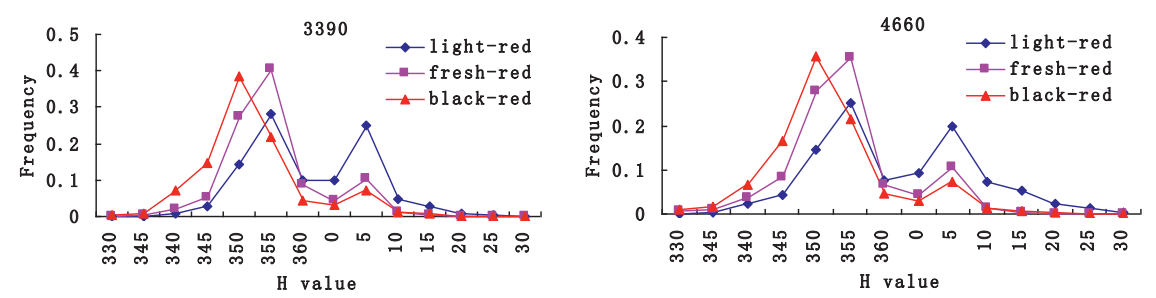

Fig. $3 \mathrm{H}$ frequency of different strawberry maturities in same light intensity

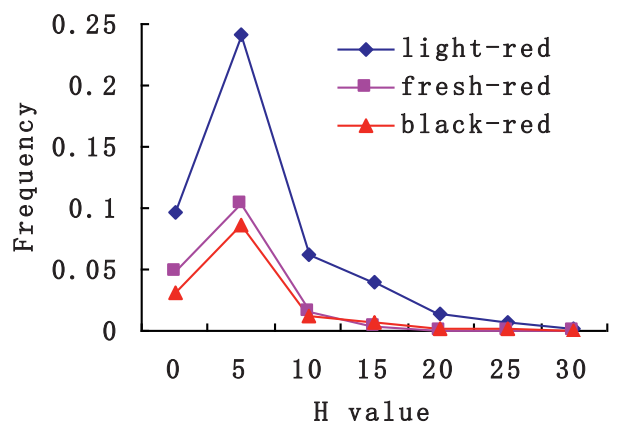

Fig. $4 \mathrm{H}$ frequency average at $\mathrm{H}=0 \sim 30^{\circ}$ of different maturity strawberry

\section{2) Chosen of colour feature value of strawberry maturity}

According to the analysis of $\mathrm{H}$ frequency of different strawberry maturities, $\mathrm{H}$ frequency is confirmed to test non-destructively strawberry maturity。 $\mathrm{H}$ is $0,5,10,15$ and 20 respectively, the change from high to low is light-red, fresh-red and black-red.

\subsection{Strawberry maturity neural network detecting system based on genetic algorithm}

The neural network is designed by using genetic algorithm, an improved genetic algorithm(Wang et al 1996) is used to optimize the structure and gain the optimum network structure.

\section{1) Structure of genetic algorithm neural network system}

To detect the strawberry maturity non-destructively, this paper constructed the strawberry maturity genetic neural network detecting system by mean of the image collecting system, the image processing system and neural network system (Fig.5). 


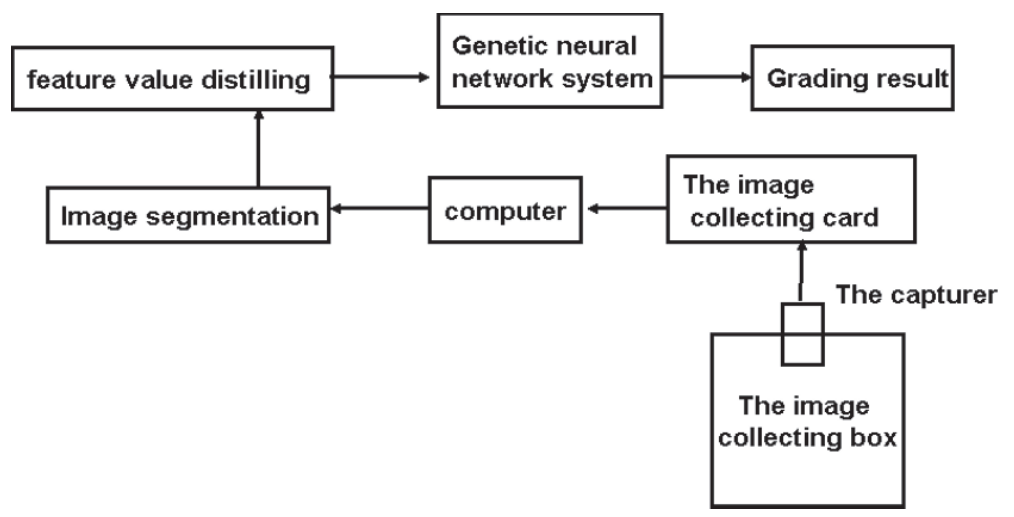

Fig. 5 The strawberry maturity neural network detecting system frame

\section{2) Design of genetic algorithm neural network system}

The design of the genetic neural network system is following:

This paper adopted the most common structure: triple-layer structures, one input layer, one hidden layer and one output layer. The network had 5 input layer nodes( $5 \mathrm{H}$ feature), and 3 output layer nodes (the maturity: black-red, fresh-red and light-red), the output layer nodes were described by a binary

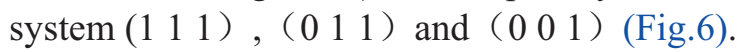

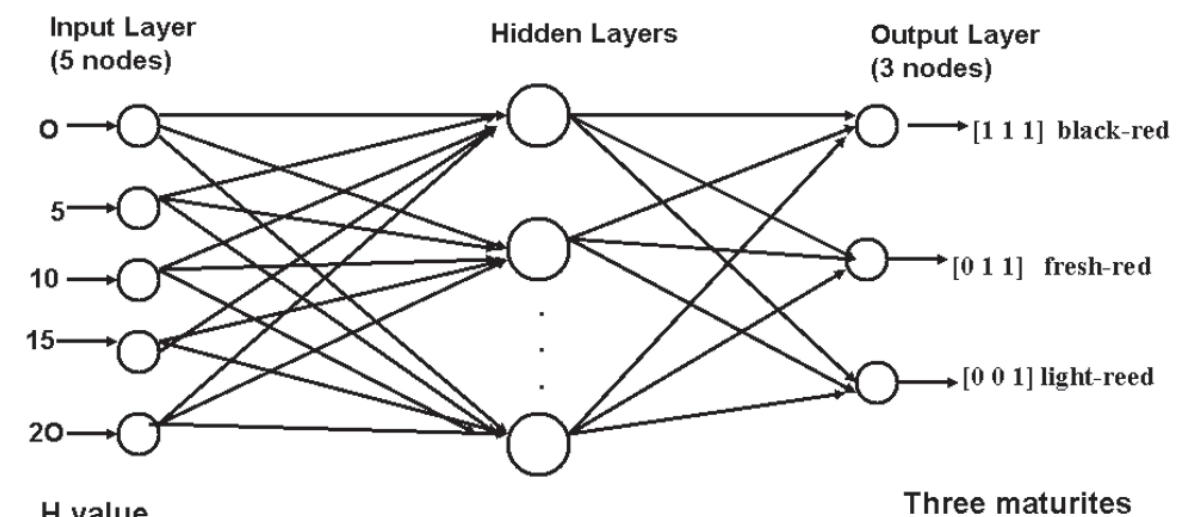

Fig. 6 The triple-layer strawberry maturity neural network detecting system

The colony scale was 20.The original number of hidden-layer node was 3 , the largest number of hidden-layer node was 13 . The crossing probability $\mathrm{P}_{\mathrm{c}}$ was 0.05 , the variance probability $\mathrm{P}_{\mathrm{m}}$ was 0.001 , the terminating condition $\mathrm{f} \leq 0.05$. 


\section{RESULTS AND DISCUSSION}

The different mature strawberries were picked from Beijing Xiaotangshan Maizhuang Strawberry Production Base, and classified into three maturities by manual according to the surface colour, twenty exemplars in every maturity were used to be trained. After the strawberry maturity genetic neural network detecting system was studied and designed, the optimum number of the hidden layer node was 7 . Therefore, the structure of neural network was 5-7-3, the training numbers were 1198, the training time was $154 \mathrm{~s}$. The test to detect strawberry maturity by using the trained neural network was completed, the training exemplars were 60 strawberries, every maturity had 20 strawberries, the test results were showed in Table 1.

The total detecting precision ratio is $91.7 \%$, the time to classify the every strawberry is $120 \mathrm{~ms}$. The collecting image time is $40 \mathrm{~ms}$, so it takes $160 \mathrm{~ms}$ to detect a strawberry. Therefore, the online detecting the strawberry maturity has been realized.

The major reason not to detect precision was that the colour of the fruit surface is not uniform.

Table 1 The result of strawberry maturity trial by genetic neural network distinguishing system

\begin{tabular}{ccccc}
\hline \multirow{2}{*}{ grade by manual } & \multicolumn{3}{c}{ Grade by mechanical system } & $\begin{array}{c}\text { detecting precision } \\
\text { ratio }(\%)\end{array}$ \\
\cline { 2 - 4 } & Black-red & Fresh-red & Light-red & 90 \\
Black-red & 18 & 2 & & 90 \\
Fresh-red & & 18 & 2 & 95 \\
Light-red & & 1 & 19 & \\
\hline
\end{tabular}

\section{CONCLUSION}

The quick and non-detective detection of agriculture product is one of the measuremeant to increase the precision and productivity of harvesting and grading. The strawberry maturity neural network detecting system based on genetic algorithm was completed. Specifically:

1) Under the different light intensities, the change of $H$ frequency of different strawberry maturities was analyzed. The $\mathrm{H}$ frequency in same strawberry maturity is not affected by the light intensities. Under the same light intensity, the change of different strawberry maturities is in order, Therefore, the section of $\mathrm{H}$ frequency is confirmed to distinguish the strawberry maturity.

2) According to the $H$ frequency, the strawberry maturity neural network detecting system was conducted. In order to decrease the training time, the genetic algorithm was adopted, the number of hidden layer node of triple- 
layer feed-forward neural network was optimized, so the training time is shorted. The results show that the total detecting precision ratio of the system is $91.7 \%$, it takes $160 \mathrm{~ms}$ to detect a strawberry. Therefore, the online detecting the strawberry maturity has been realized.

\section{REFERENCES}

B K Miller, Michael J.Delwiche. A Colour Vision System for Peach Grading. Transactions of the ASAE , 1989,32(4):1484-1490

Cao Qixin, Liu Chengliang, Yin Yuehong, et al.Colour Image Processing Based on Quality Feature Extraction of Tomato.Robt, 2001,23(7):652-656

Choi K, Lee G, Han Y J, et al. Tomato maturity evaluation using colour image analysis.Transaction of the ASAE, 1995,38(1):171-176

Mao Hanping,XU Gui li,LI Ping ping. Study on Application of Genetic Algorithm to Feature Selection of Leaves Image for Diagnosing Vegetable Disease of Nutrient Deficiency. Journal of Jiangsu University of Science and Technology, 2003,24(2):1-5

Masaru TOKUDA,Tsuneo KAWAMURA.Deveolpment of Visual System for Watermelon Harvesting Robot(Part2). Journal of Japanese Society of Agricultural Machinery, 1997,59(4):47-52

Masateru NAGATA. Study on Product Quality Estimation based on image process. Study report,March,2000

Qiu W,Shearer S A. Maturity assessment of broccoli using the discrete Fourier Transform. Transactions of the ASAE, 1992,35(6):2057-2062

Wang Qiang, Shao Huih. Genetic Evoloved Neural Network and its Application in Formaldehyde Process Modeling and Optimization. Journal of Shanghai Jiaotong University, 1996,30(4):143-150

Wang Shumao,JiaoQunying,Ji Junjie.An Impulse Response Method of Nondestructive Inspection of the Ripeness of Watermelon. Transaction of the CSAE , 1999,15(3):241-245

$\mathrm{Xu}$ Liming,Zhang Tiezhong.Influence of light intensity on extracted colour feature of different mature strawberry.New Zealand Journal of Agricultural Research. 2007,50:559565

Xu Zhenggang.Investigation of Non-destructive Citrus Maturity Determining Method Based on Image Information.Unpublished Master thesis.Zhe Jiang University,June,2001

Yang Xiukun, Chen Xiaoguang, Ma Chenglin, et al. Study on Automated Colour Inspection of Apples Using Genetic Neural Network. Transaction of the CSAE, 1997,40:173-176

Ye Qizheng,Yao Honglin,Li LI, et al.a Method for Measure Maturity According to the Feature Frequency of Resistance of Post-Harvest Fruit.Plant Physiology Communications. 1999,35(4):304-307

Yoshitaka MOTONAGA, Takaharu KAMEOKA,Atsushi HASHIMOTO.Constructing Colour Image Processing System for Managing the Surface Colour of Agricultural Products.Journal of Japanese Society of Agricultural Machinery, 1997,59(3):13-21

Zhang Changli,Fang Junlong,Fan Wei.Automated Identification of Tomato Maturation Using Multilayer Feedforward Nural Network with Genetic Alorithms(GA).Transaction of the CSAE , 2001,17(3):153-156

Zhao Jie-wen; Zou Xiao-bo; Pan Yin-fei; Liu Shao-peng. Research on method of apples odorant recognition based on GA-neural network. Journal of Jiangsu University of Science and Technology, 2004,25(1):1-4 